\title{
Design of Racetrack Coils for High-Field Dipole Magnets
}

\author{
G. Sabbi, S. Caspi, S.A. Gourlay, R. Hafalia, A. Jackson, A. Lietzke, A.D. McInturff, R.M. Scanlan
}

\begin{abstract}
The magnet group at LBNL is currently in the process of developing high-field accelerator magnets for use in future colliders. One of the primary challenges is to provide a design which is cost-effective and simple to manufacture, at the same time resulting in good training performance and field quality adequate for accelerator operation. Recent studies have focused on a racetrack geometry that has the virtues of simplicity and conductor compatibility. The results have been applied to the design of a series of prototype high-field magnets based on $\mathrm{Nb}_{3} \mathrm{Sn}$ conductor.
\end{abstract}

\section{INTRODUCTION}

A program to develop high-field accelerator magnets for future colliders is underway at several U.S. laboratories. After completion of the $13.5 \mathrm{~T} \cos -\theta$ dipole $\mathrm{D}-20$ [1], the LBNL magnet group has started to investigate a recently proposed "common coil" configuration for two-aperture dipoles, using racetrack coils shared between both magnet apertures [2] [3]. The first test involved a $6 \mathrm{~T}$ short model (RD-2) built using ITER-type $\mathrm{Nb}_{3} \mathrm{Sn}$ conductor. The magnet reached short sample field with no training in a variety of configurations [4]. The following magnet in this series (RD-3) uses high-performance $\mathrm{Nb}_{3} \mathrm{Sn}$ conductor with critical current density above $2 \mathrm{kA} / \mathrm{mm}^{2}$ at $12 \mathrm{~T}, 4.2 \mathrm{~K}$, and is designed to reach $14 \mathrm{~T}$ [5]. The coil structure is composed of two outer modules and one inner module. The two outer modules have been pre-tested in back-to-back configuration (RT-1) and have achieved a field of $12 \mathrm{~T}$ [6]. The complete structure is now assembled and testing is underway.

Present experiments are focused on basic structural issues and the development of fabrication techniques for the new configuration. Goal for the next phase of the program is to achieve field quality suitable for operation in high-energy colliders while retaining the highest level of simplicity and efficiency. This paper presents a list of requirements for the experimental program, and describes a series of prototype magnets which are being designed to satisfy these requirements.

Manuscript received September 18, 2000.

Authors are with Lawrence Berkeley National Laboratory, One Cyclotron Road, Berkeley, CA 94720.

Work supported by the U.S. Department of Energy.

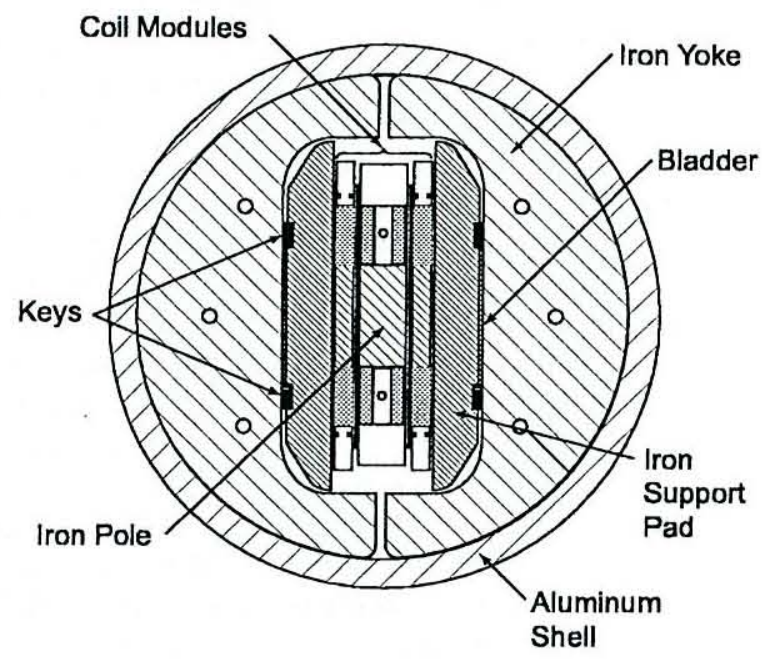

Fig. 1. RD-3 cross-section.

\section{Design Requirements}

Fig. 1 shows a cross-section of the RD-3 dipole. The magnet has three major components. The first component comprises 3 coil modules wound around iron cores (islands). For each module, vertical pre-load is provided by face plates (skins) welded to side rails. The second component is the iron yoke, which serves as a flux return. The third component is the outer aluminum shell, which provides support against the large horizontal forces. The yoke is split between an inner pad and an external structure. Keys are inserted in the resulting gap using a Pressurized Bladder System (PBS) to reach the desired prestress at room temperature [7]. Two bore pieces, each one providing a $10 \mathrm{~mm}$ clear aperture, are inserted between the coils.

The first requirement for the next phase of the magnet program is to increase the clear bore diameter from $10 \mathrm{~mm}$ to $40 \mathrm{~mm}$. It should be noted that significant cost savings can be realized by decreasing the magnet aperture of future colliders to lower values than in present machines. The racetrack configuration is particularly well suited to fabrication of small-aperture dipoles. Preliminary studies indicate that a racetrack magnet with coil to coil spacing of $20 \mathrm{~mm}$ can be fabricated, using $0.7 \mathrm{~mm}$ strand, with all harmonics well within one unit within a circle of $14 \mathrm{~mm}$ diameter (2/3 of the coil to coil spacing) [8]. However, it is first necessary to demonstrate that a high-field 
racetrack dipole can be built with sufficiently large bore to meet present accelerator technology standards. The main challenge is providing adequate coil support against the large horizontal pre-load required to prevent coil displacement during excitation. The horizontal pre-load is reacted by iron islands and bore spacers. Since the thickness of the bore spacer needs to be minimized for conductor efficiency, it is desirable to react most of the horizontal pre-load against the iron island. This technique however, requires tight tolerances to be met in magnet fabrication. One can also partly rely on rigidity of the (impregnated) coils for horizontal support. Determination of the minimum thickness of the bore spacer is one of the goals of the experimental program. For improved conductor efficiency, the skin thickness should also be minimized. With respect to the present value of $4.75 \mathrm{~mm}$, it is planned to significantly reduce the skin thickness in new magnets, or even eliminate the skin altogether and rely on lateral friction after final assembly to provide vertical support. A technology development program using mechanical models is underway to address these and other issues.

The second requirement for the experimental program is to achieve adequate field quality for operation in highenergy colliders. This typically translates to field errors within one part in $10^{4}$ ("unit") at the maximum radius where stable particle motion is required. It has been shown that in small-aperture, moderate field (10-11 T) racetrack dipoles, adequate field quality can be obtained using solely the main coils [9]. However, for a high-field racetrack magnet with $40 \mathrm{~mm}$ clear bore, conductor efficiency dictates that "auxiliary" condutor blocks be placed in the vicinity of the pole to minimize the geometric harmonics. Resulting mechanical issues are the need to provide vertical support for the auxiliary turns and the need to return these conductors at the magnet ends without interfering with the beam tube. Two approaches have been investigated, resulting in a departure from either the planar coil geometry or the large bending radius feature of the common coil layout.

Variations of the harmonics during excitation should also be limited to a few units. The distance between the two apertures and the profiles of iron islands and yoke have to be optimized to achieve low saturation harmonics. Use of saturation control cut-outs can only be applied to a limited extent in this case due to role of the yoke in providing mechanical support to the coil modules.
Present high current density $\mathrm{Nb}_{3} \mathrm{Sn}$ conductors are limited to an effective filament size of about $80 \mu \mathrm{m}$, leading to significant field distortions at low excitation due to persistent currents. Compensation of persistent current effects by iron yoke design or use of magnetic inserts [9] [10] will be explored to improve the injection field quality and increase the dynamic range. At the same time, a conductor development program presently underway has among its goals a $50 \%$ reduction of the effective filament size [11].

\section{RD-4 MAGNET}

As a first step towards fabrication of a racetrack dipole with collider-class field quality, it is planned to reassemble the RD-3 outer coils, together with two newly fabricated inner coils, in a magnet with field above $13 \mathrm{~T}$ and $35 \mathrm{~mm}$ clear bore. Fig. 2 shows the reference coil cross-section for one aperture, optimized using ROXIE [12]. Each of the new inner coils is a 2-layer double pancake, where the inner layer has the role of auxiliary coil for field quality adjustment. Two 9-turn blocks are located at opposite locations close to the pole, supported by the bore spacer. The cable design is the same as in the inner layer of RD-3 (40 strands, $0.8 \mathrm{~mm}$ diameter).

An alternate coil cross-section is shown in Fig. 3. In this case, the auxiliary turns are wound separately from the main inner coil, in a left-right geometry. An important advantage of this configuration is that the auxiliary coil cable can be graded to obtain better conductor efficiency (in this case, the auxiliary cable uses 44 strands instead than 40). Another advantage is that a planar racetrack geometry can be maintained at the coil ends. However, this configuration requires a larger number of coils (and joints) and is more complicated to assemble. Also, the winding radius for the auxiliary coil modules is not significantly larger than in a $\cos -\theta$ dipole, preventing the possibility of winding this type of coil using reacted cable.

Table I shows the calculated short sample parameters in the magnet body for the two coil design options, based on strand $I_{c}$ measurements. No cabling degradation is expected, consistent with the results of the RT-1 test. The calculation assumes an iron yoke configuration very similar to RD-3 (Fig. 1) except for a $2 \mathrm{~cm}$ shift outwards of the boundary between coil module and yoke. The short sample field for design option $\mathrm{B}$ is higher by $0.4 \mathrm{~T}$. This
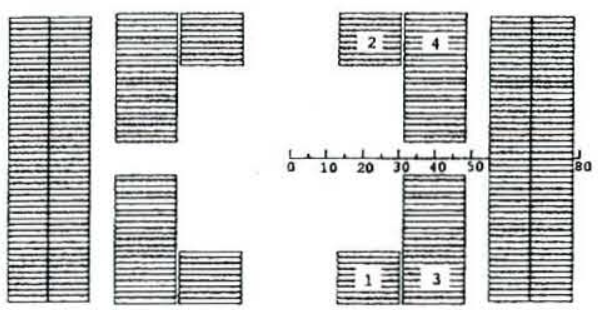

Fig. 2. RD-4 coil cross-section "A" (one aperture).

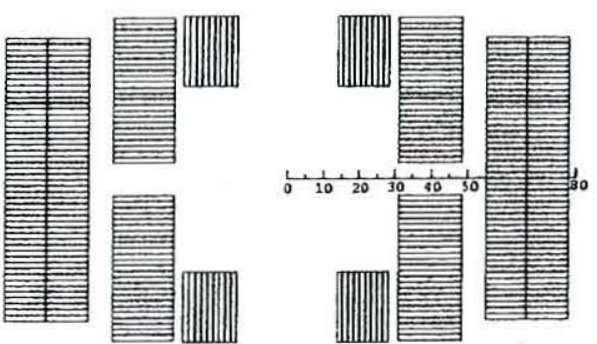

Fig. 3. RD-4 coil cross-section "B" (one aperture). 
TABLE I

RD-4 Short SAMPle PARAMETERS (2D).

\begin{tabular}{cccc}
\hline Parameter & Unit & Design A & Design B \\
\hline Short sample dipole field & $\mathrm{T}$ & 13.4 & 13.8 \\
Max. current (inner coil) & $\mathrm{kA}$ & 13.2 & 13.1 \\
Max. current (aux. coil) & $\mathrm{kA}$ & 12.9 & 13.2 \\
Max. current (outer coil) & $\mathrm{kA}$ & 13.1 & 13.4 \\
\hline
\end{tabular}

is due to grading of the cable in the auxiliary turns and a $7 \%$ increase in coil area. Design A has been chosen as reference because it allows use of double pancake coils in the inner module. We are interested in developing this type of inner module as a possible candidate for a moderate field, cost-effective dipole for VLHC [13].

Both candidate coil configurations for RD-4 can provide geometric harmonics within a fraction of a unit at a reference radius of $10 \mathrm{~mm}$. However, the decision to use the RD-3 outer coil modules (in order to provide faster turnaround) makes it difficult to achieve satisfactory saturation harmonics. The distance between the 2 apertures in RD-3 is $220 \mathrm{~mm}$. This value was set in part due to fabrication constraints (size of the coil package) and is adequate for the goals of the RD-3 experiment. However, a larger aperture separation (at least $300 \mathrm{~mm}$ ) would be desirable to control saturation harmonics while preserving high dipole field in RD-4. Fig. 4 shows the RD-4 saturation harmonics for two different configurations. In the first case (R70) both the inner and the outer coil islands are made entirely of iron, up to the $70 \mathrm{~mm}$ minimum coil winding radius, as in the RD-3 magnet. In the second configuration (R35) the inner module island is made of iron from the magnet center up to $35 \mathrm{~mm}$, while non magnetic material (for example $\mathrm{AlCu}$ ) is used from $35 \mathrm{~mm}$ to $70 \mathrm{~mm}$. As can be seen, large field errors appear in the first configuration due to off-centering of the coil package with respect to the yoke. The normal sextupole starts at -35 units and approaches zero during magnet excitation, while a positive sextupole at low current is required for compensation of field errors due to conductor magnetization. A very large variation of the skew quadrupole is also observed. The second configuration has the desired sextupole characteristics, and the skew quadrupole variation is reduced, but the short sample dipole field is lower by
$0.5 \mathrm{~T}$ due to insufficient iron thickness in the central region of the magnet to shield the opposing fields generated in the two apertures. Considering that the first configuration has relatively small saturation harmonics at nominal field, it was decided to adopt it for RD-4 and address the saturation control issue at a later stage in the program.

The end configuration for the reference design is shown in Fig. 5. While block 1 (Fig. 2) can be wound flat without interfering with the bore, block 2 is hard-way bent outwards in an S-shape before being returned on top of block 4 . The resulting longitudinal displacement of block 2 with respect to the other conductor groups is also beneficial in order to compensate the intrinsic end asymmetry of the racetrack configuration and achieve low integrated end harmonics. End design and optimization is in progress. Use of the RD-3 outer modules, together with constraints on the total length of the magnet, limits the possibility to carry out end field quality optimization in RD-4. It would be desirable to extend the length of the inner module further than the outer, but the length of the outer module is already close to the maximum allowed by the test facility. For the same reason, it may be difficult to optimize the end layout to achieve low peak field, resulting in some reduction of the short sample dipole field with respect to the value obtained for the magnet body.

The RD-4 experiment will develop a simple coil structure capable of good field quality, address the issue of internal coil support and improve conductor efficiency with respect to previous models by reducing the skin thickness in the inner coils. It is expected to achieve field errors within one part in $10^{3}$ at nominal current, and to start addressing fabrication issues in order to improve the accuracy and predictability of conductor placement.

\section{RD-5 MAGNET}

Design goals for RD-5 are an increase of the dipole field to $14 \mathrm{~T}$, an increase of the clear bore size to $40 \mathrm{~mm}$ and a reduction of the saturation harmonics with respect to RD-4. Fig. 6 shows a preliminary cross-section geometry. The coil design is similar to RD-4, but a few more turns are used in each layer and a midplane spacer is added in

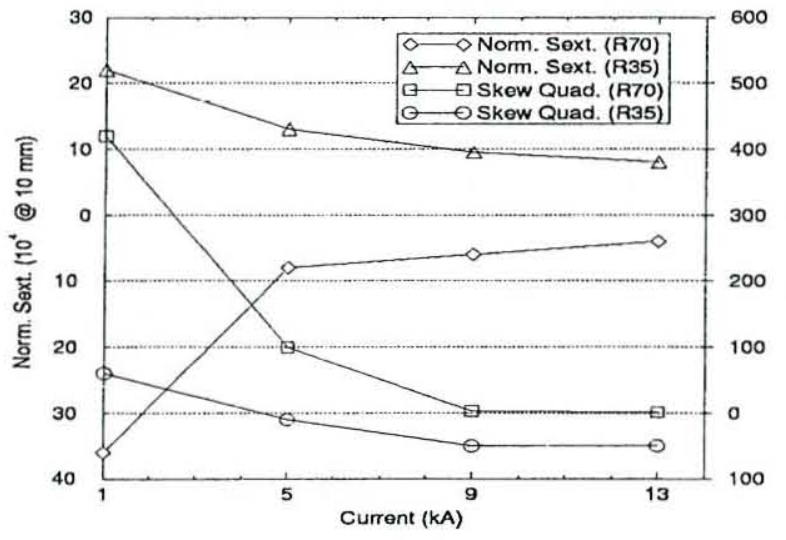

Fig. 4. RD-4 saturation harmonics.

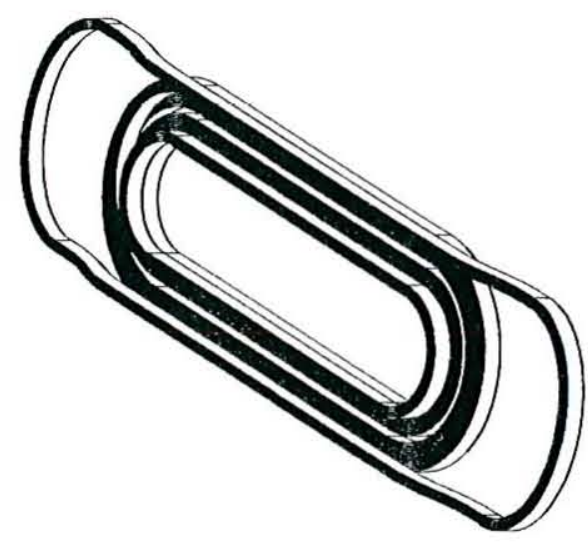

Fig. 5. Coil layout for RD-4-A. 


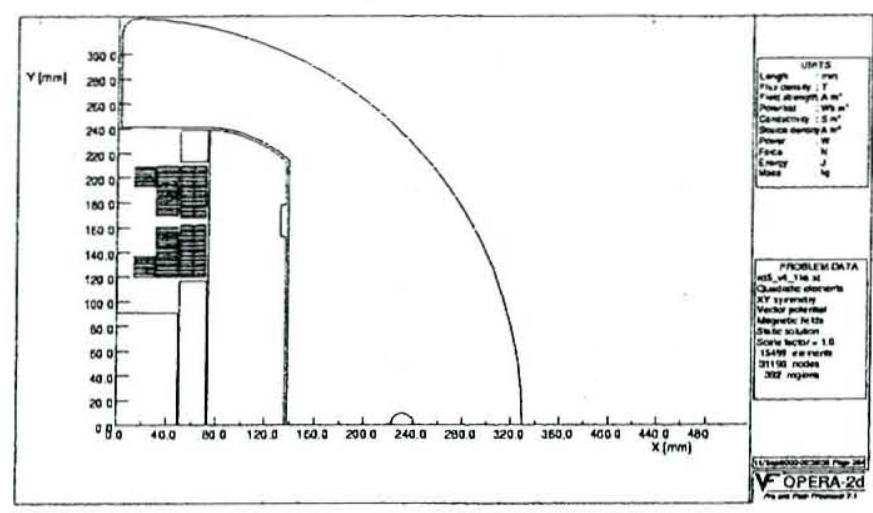

Fig. 6. RD-5 cross-section.

the outer coil. For improved efficiency, a thin skin (or no skin) is used in both layers and the outer cable is narrower (24 strands instead than 26). This also allows to bring the iron yoke closer to the bore. The short sample field based on critical current measurements of available inner and outer strand is $14 \mathrm{~T}$ at $11.6 \mathrm{kA}$. A dipole field of $15 \mathrm{~T}$ can be reached with an increase of the $\mathrm{Nb}_{3} \mathrm{Sn}$ critical current density to $3 \mathrm{kA}$ at $12 \mathrm{~T}, 4.2 \mathrm{~K}$. This is one of the goals of the conductor development program [11].

In order to improve the saturation harmonics with respect to RD-4 without compromising the dipole field, the distance between apertures is increased from $220 \mathrm{~mm}$ to $330 \mathrm{~mm}$. The central island of the inner layer is split between a magnetic core and a nonmagnetic perimeter. Both the island and the side rail for the outer module are made of iron.

Fig. 7 shows the RD-5 saturation harmonics. The normal sextupole is positive at low current, allowing some compensation of the field distortion due to conductor magnetization. Compensation of the persistent current decapole can be achieved by placing a magnetic strip at the location of the inner coil midplane spacer. A positive bump of both the normal sextupole and the skew quadrupole is observed at mid-range excitation. This effect is not present if a smoother profile is used for the yoke and island [8] [9], but the structure becomes more complicated. Evaluation of the impact of these errors on beam dynamics would be useful to guide further yoke optimization.

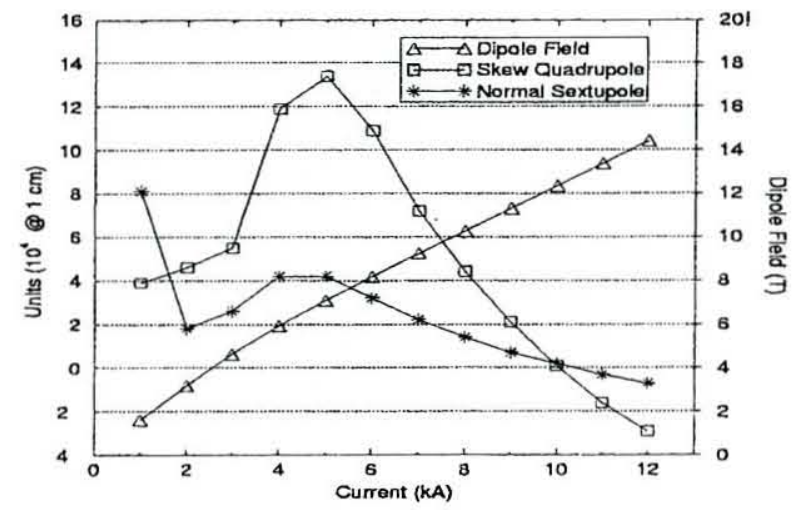

Fig. 7. RD-5 saturation harmonics.
TABLE II

RD-5 Straight Section Harmonics (10 kA).

\begin{tabular}{cccc}
\hline Normal & Units @ $10 \mathrm{~mm}$ & Skew & Units @ $10 \mathrm{~mm}$ \\
\hline $\mathrm{b}_{3}$ & 0.18 & $\mathrm{a}_{2}$ & 0.10 \\
$\mathrm{~b}_{5}$ & 0.05 & $\mathrm{a}_{4}$ & -0.02 \\
$\mathrm{~b}_{7}$ & -0.05 & $\mathrm{a}_{6}$ & $<0.01$ \\
$\mathrm{~b}_{9}$ & -0.02 & $\mathrm{a}_{8}$ & $<0.01$ \\
\hline
\end{tabular}

The RD-5 field errors at nominal current $(10 \mathrm{kA}, 85 \%$ of short sample) are shown in Table II. All design harmonics are very small, providing a good reference for model magnet fabrication and testing.

\section{CONCLUSIONS}

High-field, racetrack dipoles are being developed at LBNL for use in future colliders. Goal for the next phase of the program is to reach adequate field quality for collider operation, while preserving the highest level of simplicity and efficiency. The main features of 2 model magnets which are being designed to address these issues have been presented.

\section{REFERENCES}

[1] A.D. McInturff et al., "Test Results for a High Field (13 T) $\mathrm{Nb}_{3} \mathrm{Sn}$ Dipole", 1997 Particle Accelerator Conf., Vancouver, May 1997.

[2] R. Gupta, "A Common Coil design for High-Field 2-in-1 Accelerator Magnets", 1997 Particle Accelerator Conference, Vancouver, May 1997.

[3] R. Gupta, "Field Quality Optimization in a Common Coil Magnet Design", IEEE Trans. Appl. Supercond. Vol. 10 No. 1 (March 2000), pp. 326-329.

[4] S. A. Gourlay et al., "Fabrication and Test Results of a Prototype, $\mathrm{Nb}_{3} \mathrm{Sn}$ Superconducting Racetrack Dipole Magnet" ${ }^{n}, 1999$ Particle Accelerator Conf., New York, March 1999.

[5] K. Chow et al., "Mechanical Design of a High Field Common Coil Magnet", 1999 Particle Accelerator Conf., New York, March 1999.

[6] A. Lietzke, "Racetrack Coil Technology Validation (RT-1) Test Results", Proceedings of the 2nd VLHC Magnet Workshop, Fermilab, May 2000 (bttp://vlhc.org).

[7] S. Caspi et al., "The Use of Pressurized Bladders for Stress Control of Superconducting Magnets" Proceedings of this Conference.

[8] G. Sabbi, "Fermilab Common Coil Magnet Development", Proceedings of the 2nd VLHC Magnet Workshop, Fermilab, May 2000 (http://vlhc.org).

[9] G. Sabbi et al., "Conceptual Design of a Common Coil Dipole for VLHC", IEEE Trans. Appl. Supercond. Vol. 10 No. 1 (March 2000), pp. 330-333.

[10] S. Caspi, "Reduction of Magnetization Induced Harmonics in Superconducting Magnets", LBNL SC-MAG-691, October 1999.

[11] R. Scanlan, " $\mathrm{Nb}_{3} \mathrm{Sn}$ Conductor Development for High Energy Physics-Plans and Status", Proceedings of this Conference.

[12] S. Russenschuck et al., "Integrated Design of Superconducting Accelerator Magnets - a Case Study of the Main Quadrupole", The European Physical Journal (Applied Physics), p.93-102, January 1998.

[13] G. Dugan, "Very Large Hadron Collider R\&D", 1999 Particle Accelerator Conference, New York, April 1999. 